\title{
State of the Art in Stratum Corneum Research. Part II: Hypothetical Stratum Corneum Lipid Matrix Models
}

\author{
Thomas Schmitt $^{\mathrm{a}}$ Reinhard H.H. Neubert ${ }^{\mathrm{b}, \mathrm{c}}$ \\ aDepartment I, Institute of Anatomy and Cell Biology, Ludwig-Maximilians-Universität München, Munich, Germany; \\ bInstitute of Applied Dermatopharmacy at the Martin Luther University Halle-Wittenberg (IADP), \\ Halle/Saale, Germany; 'Institute of Pharmacy, Martin Luther University Halle-Wittenberg, Halle/Saale, Germany
}

\section{Keywords}

Ceramides · Stratum corneum - Hypothetical models ·

Lipid model systems · Biophysics

\begin{abstract}
This review is the second part of a series which presents the state of the art in stratum corneum (SC) lipid matrix (LM) research in depth. In this part, the various hypothetical models which were developed to describe the structure and function of the SC LM as the skin's barrier will be discussed. New as well as a cumulative assortment of older results which change the view on the different models are considered to conclude how well the different models are holding up today. As a final conclusion, a model, factoring in as much of the known data as possible, is concluded, unifying the varying different models into one which can be developed further, as new results are found in the future. So far, the model is described with a single crystalline or gel-like phase with a certain amount of nanocrystallites of concentrated ceramides (CERs) and free fatty acids and more fluid nanodomains caused by a fluidizing effect of the cholesterol. These domains are dynamically resolved and reformed and do not impair the barrier function. The chain conformation is not
\end{abstract}

karger@karger.com www.karger.com/spp

Karger $\stackrel{\text { ' }}{5}$

GOPEN ACCESS
(C) 2020 The Author(s)

Published by S. Karger AG, Basel

This is an Open Access article licensed under the Creative Commons Attribution-NonCommercial-4.0 International License (CC BY-NC) (http://www.karger.com/Services/OpenAccessLicense), applicable to the online version of the article only. Usage and distribution for commercial purposes requires written permission. completely clear yet; however, an equilibrium of fully extended and hairpin-folded CERs with ratios depending on the properties of each individual CER species is proposed as most likely. An overlapping middle layer as described for the tri-layer model in part I of this series would be present for both conformations. The macroscopic broad-narrow-broad layering, observed in electron micrographs, is explained by an external templating by the lipid envelope, and an internal templating by short and long lipid chains each preferentially show a homophilic association, forming thicker and thinner bilayers, respectively. The degree of influence of the very long $\omega$-hydroxy-CERs is discussed as well.

(C) 2020 The Author(s)

Published by S. Karger AG, Basel

\section{Introduction}

The stratum corneum (SC) is the outermost layer of the mammalian skin. As such, it represents the body's main interface with its environment. It provides a strong penetration barrier protecting the body from harmful influences like pathogens or exogenous chemicals. It also limits the water loss of the skin and is, thus, very important for all forms of terrestrial life. In the 1960s, penetra- 
tion experiments demonstrated that this penetration barrier of the skin is located within the SC $[1,2]$. This skin layer is constructed from dead cells without a nucleus and a regular cell membrane, the corneocytes (CORs). These cells are heavily cross-linked via corneodesmosomes [3] forming a strong rigid tissue. The CORs are also heavily keratinized and surrounded by a mantle of proteins and lipids, the corneocyte envelope (CE). This multilayered shell replaces the cellular membrane in this terminal stage of differentiation. It consists of an inner layer of crosslinked proteins bound to the keratin network, the cornified protein envelope [4]. A monolayer of long $\omega$-hydroxyceramides ( $\omega$-OH-CERs), the cornified lipid envelope (CLE), is covalently bound to the surface of the cornified envelope [5-7]. A complex free multi-lamellar arranged lipid matrix (LM) is filling the remaining free intercellular space. This matrix consists of ceramides (CERs), free fatty acids (FFAs) and cholesterol (CHOL) in an about equimolar ratio [8]. The LM was reported to almost exclusively be responsible for the effectiveness of the skin barrier [9]. The CERs as most complex of these lipids determine the overall phase behaviour and nanostructure of the LM [10-12] and consequently are most important for the barrier function [4]. Part I of this review series already discussed the general biophysical properties of the different CER species in detail [13]. In this part, different models trying to describe the LM structure will be highlighted and discussed. The new results shown in part I as well as a cumulative assessment of further important factors which either consent or contradict a certain model will be considered.

\section{The Brick and Mortar Model}

The most basic description of the overall SC is the brick and mortar model first proposed by Michaels et al. [14] and later supported by Elias [15, 16]. This model describes the basic structure of the overall SC depicting it as a brick wall. The cell bodies represent the bricks and the LM and the mortar in between them. The lipid molecules within the LM according to a pure stochastic viewpoint were assumed to be arranged in simple bilayers [14-16]. However, the lamellar microstructure and the molecular nanostructure were soon found to be much more complex than anticipated. Considering what we know about the SC today, the brick and mortar model is preliminary and is not giving a sufficient representation of the properties of the SC. In this model, the CORs are not connected, and they are relatively loosely arranged by van der Waals interactions and hydrogen bonds with the CLE as its only real anchor [5-7]. Even without the LM, the CORs are nothing like loose bricks but are tightly connected via the corneodesmosomes [3]. The LM cannot be described as a permeable mortar but actually holds the main penetration barrier [9], while the CORs unlike bricks are rather permeable in comparison [17]. This leaves the brick and mortar model as a relatively rudimentary depiction of the macroscopic structure of the SC. Soon, more intensive investigations on the structure and function of the SC and, especially, the LM followed. As a result of these various differing experimental observations, a number of different models trying to describe the architecture of the LM in more detail were brought forward, some of them being very similar and others completely contradictory to each other. All of these models are, however, still controversially discussed within the scientific community even today.

The review by Kessner et al. [18] last approached the controversial topic comparing the different models and assessing the state of the art. Even though some features are yet unclear, many new results have been produced since then, providing various new insights.

\section{General Aspects of the LM Organization}

From freeze fracture electron microscopic experiments in the 1970s, it was discovered that the SC LM had a multi-lamellar organization [19] consisting of mostly 3 , 6 , or 9 consecutive lamellae [20]. With the utilization of $\mathrm{RuO}_{4}$, it became possible to visualize the intercellular lamellae in electron micrographs. The strongly stained head groups appeared as electron-dense bands, while the acyl chains appeared as electron-lucent bands. It was observed that the lamellae are arranged approximately parallel to the cell surface [21-25]. It could be shown that the LM had not only one but at least 2 distinctly different lamellar arrangements. The 11-14-nm [21, 22] long periodicity phase (LPP) and the 5-6.5-nm [13, 26-28] short periodicity phase (SPP), sometimes also $4-4.5 \mathrm{~nm}[29$, 30] thick, lamellae are described. Because the LPP is a very controversial and broad topic all on its own, the exact structure of the LPP is yet unknown, and different studies often use completely differently composed model systems. This often generates varying or even contradicting results. Many studies do not detect an LPP at all [31]. While in skin, an LPP is usually observed and shows a repeat distance of about $14.5 \mathrm{~nm}$ [17, 32-38]. In model systems, it can often only be detected if the ratio of $\omega-\mathrm{OH}$ CERs is much higher than in the native skin and its repeat distance with $13 \mathrm{~nm}$ is slightly different [39-48]. This poses the question that if any of the observed arrangements are comparable to the LPP in the native skin. How- 


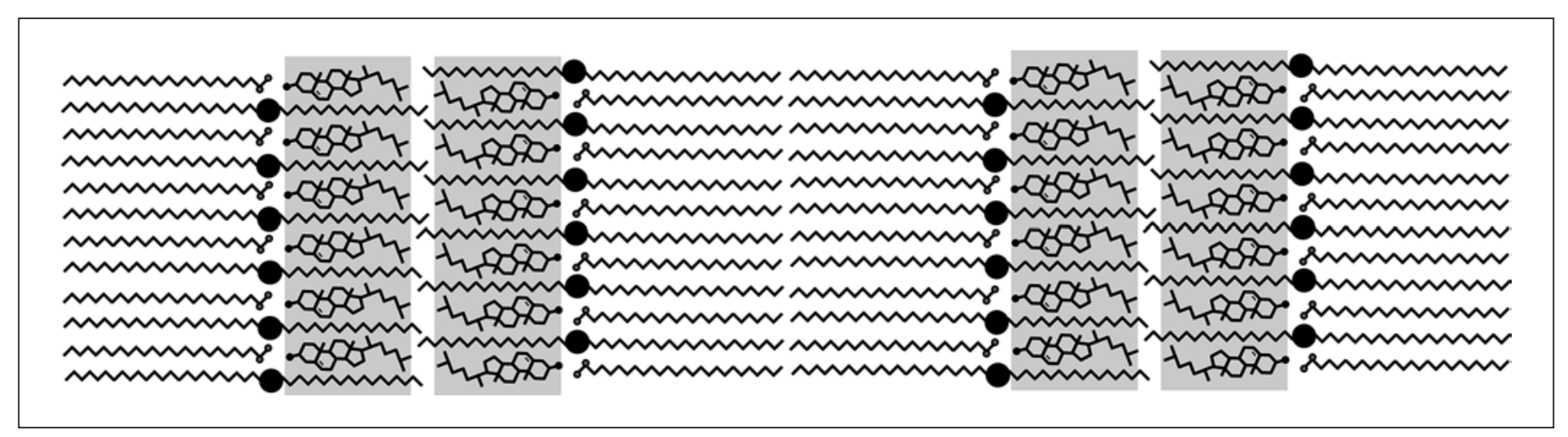

Fig. 1. Schematic representation of the LM architecture according to the asymmetry model. LM, lipid matrix.

ever, this question is a topic broad enough for an own review all. This review will, thus, focus only on the much better studied and described structure of the SPP. Most of the CERs are asymmetric > C20 CERs, but a certain pool of symmetric short chain C16-C18 is present [49], representing an "island of stability." The shorter CERs were used in most of the earlier studies, influencing some of the hypothetical models and the properties they propose for the LM [50,51]. The CERs of 2 chains can assume 3 different basic conformations, depending on the chain opening angle $\gamma$, a hairpin conformation with $\gamma=0^{\circ}$, a fully extended conformation with $\gamma=180^{\circ}$, and a more or less V-shaped conformation for all angles in between. Due to the technical limitations of the used methods so far, it is still unclear which conformation is favoured or if different CERs possibly even favour several different conformations to a different degree. So far, only one of the 2 chains was deuterated, which made it impossible to definitely conclude the conformation of the second chain, since small differences in the deuteration position can also originate from chain tilts, chain disorder, or degree of chain overlapping. Deuterating both chains of the lipids and using several variants with the 2 deuterations in different positions would make it possible to identify each chain and reconstruct the exact conformation. Especially, if these data are evaluated, using highly detailed molecular dynamics simulations (MDS), it could be possible to finally learn about the chain conformation. CERs can further yield one of 3 lateral packings the dense crystallinelike orthorhombic one, the gel-like hexagonal one, and the loose liquid-like one [52].

\section{The Asymmetry (Splayed Bilayer) Model}

Although mainly discussed in recent years and, thus, considered a new idea [53], the concept that this model was actually the first one to be proposed out of all the models will be discussed here. This model is based on the interpretation of the specific broad-narrow-broad band pattern of alternating electron lucent and electron dense bands, observed within electron micrographs. In 1987, this pattern was at first attributed to a directed asymmetric lipid distribution. The long chains were suggested to be arranged in one sub-cell and the short chains would be arranged in the other sub-cell. The bilayers were interpreted to be made up of either 2 long or 2 short subcells with long and short bilayers alternating, resulting in the broad-narrow-broad pattern [21]. Later, this pattern was concluded to rather be the result of the influence of the very long $\omega$-OH CERs only and consequently attributed to the LPP $[17,22,32,34,35,39,40,42,54]$. In 2012 , new cryo-electron microscopy investigations of vitreous skin sections defocus series combined with atomistic 3D cryo-EM model simulations again suggested the possibility of such an arrangement which would not be solely or mainly based on the influence of the very long $\omega-\mathrm{OH}$ CERs. Using model simulations, it was estimated that the repeating broad and narrow electron-lucent bands observed in the electron micrographs would be explained best by an arrangement based on fully extended longchain CERs. The presence of isolated 5-6.5-nm and/or 4-4.5-nm units in the upper SC layers and, thus, within the functional barrier region was contradicted by these results. These isolated units are only found within the lower layers of the SC and can, thus, rather be contributed to the formation process of LM than to the mature and functional LM. In the uppermost functional barrier regions, the presence of only pairs of $5.5+4.5-\mathrm{nm}$ or 6.5 $+4.5-\mathrm{nm}$ units, forming a combined $10-11-\mathrm{nm}$ repeating unit, is suggested [55-58] (Fig. 1). It was also suggested that the CHOL would only be found in the short 


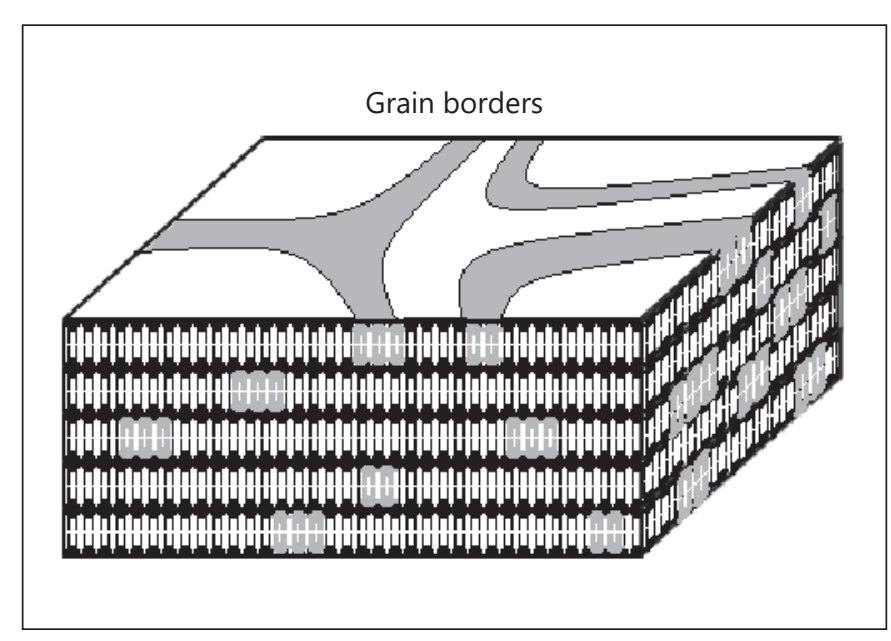

Fig. 2. Schematic representation of the LM architecture according to the domain mosaic model $[60,61]$. LM, lipid matrix.

bilayer due to its better miscibility with the short chains, while the long FFAs would only be found in the long bilayer [58]. Another study in 2014 using infrared spectroscopy also suggested a similar arrangement with the association of CHOL with the short and the long FFAs with the long chains in separate bilayer units [59]. This structure could possibly explain both the SPP and what is so far described as the LPP with the coupled 11-nm repeating unit, proposing only a single gel-like lamellar phase [55-58].

\section{The Domain Mosaic Model}

Furthermore, the domain mosaic model was described. This model is based on the original assumption of a simple bilayer arrangement but would not necessarily contradict a fully extended conformation. The novel aspect of this model, however, is an assumption about the lateral structure and lipid phase behaviour rather than the perpendicular layer arrangement. Since crystalline orthorhombic as well as more liquid characteristics were observed side by side within the LM [28], this model suggests an arrangement of the lipids into domains with differing chain packing and phase behaviour. The lamellae would consist of crystalline-like grains embedded in a continuous more liquid matrix. At the liquid-like grain boarders, the penetration resistance is reduced due to the less dense lipid packing and increased molecular mobility. This barrier disturbance is suggested to allow for a necessary minimum of transepidermal exchange of water and other substances $[60$, 61] (Fig. 2).

\section{The Sandwich Model}

A bit later, the sandwich model was introduced. This model was originally based on X-ray diffraction data on isolated human skin, which first took temperature and hydration into account. This model as shortly mentioned above reinterpreted the broad-narrow-broad arrangement to be the result of a different separate microstructural arrangement in the form of 12-13-nm LPP [17, 32]. For the SPP, a simple symmetric bilayer arrangement with mainly randomly distributed lipids in a single lateral phase was concluded $[62,63]$. Swelling and, thus, water absorption by the lamellar structure was not observed or is just very minimal. The observed liquid phase proportion was suggested to be a feature which can be solely contributed to the LPP [13,26-28]. This model is overall the most described one up to date.

\section{The Single-Gel Phase Model}

Soon, the single-gel phase model came out. This model just like the domain mosaic model describes a specific lateral and phase behaviour instead of a perpendicular arrangement. It is assumed that even though the lipids have a different orthorhombic or hexagonal chain packing, they do not form different domains. The lipids rather form a single coherent gel-like phase, without any grain borders. The water content in this arrangement would be minimal with very low overall water permeability. The lipid mobility would also be very low. The conformation of the lipids could be either a fully extended or a hairpin one since both would be possible [50,51]. It would, thus, be compatible with either the splayed bilayer or the sandwich model.

\section{The Armature Reinforcement Model}

Later on, the armature reinforcement model was presented. It is basically an extension of the sandwich model for the SPP. The outstanding difference is the assumption of a small amount of fully extended CERs which serve as a cross-linker between multiple bilayers of otherwise hairpin-folded CERs. As a consequence, they are assumed to reinforce the armature represented by the head groups cross-linking the opposing head group regions of 2 adjacent lamellar leaflets. This arrangement would give the LM additional stability [64]. This way, the model is also able to explain the occurrence of swelling upon hydration by a conformation-change mechanism. Upon hydration, the full extended conformation switches to a hairpin conformation allowing water to penetrate within the head group region, which increases the spacing [65] (Fig. 3). 


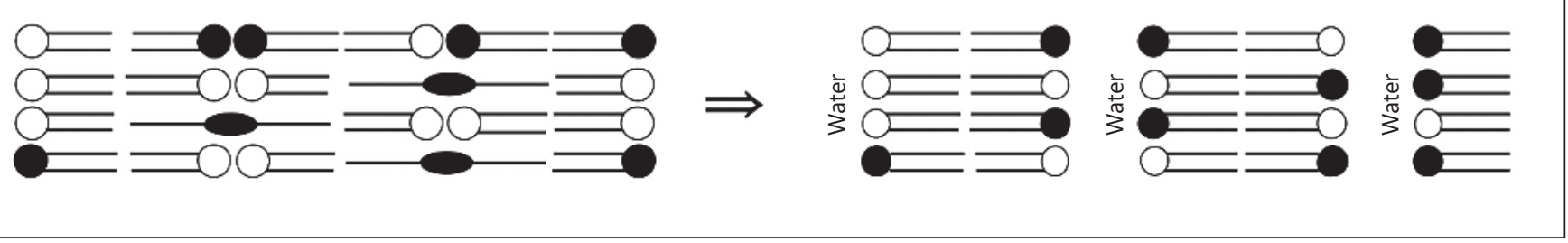

Fig. 3. Schematic representation of the LM architecture according to the armature reinforcement model. The model is shown as presented in the original publication by Kiselev et al. [65], including some simplifications (not showing the homogeneously distributed FFAs and CHOL). LM, lipid matrix; FFAs, free fatty acids; CHOL, cholesterol.

\section{The Tri-Layer Model}

In part I of this review series [13], a new hypothetical model describing the nanostructural arrangement of the LM lipids based on new results on the properties of CERs was introduced. This model can also be considered an extension or update of the sandwich model. It is assuming an arrangement of multiple symmetrical bilayers. It is, however, not necessarily contradicting an arrangement completely or partially made up of fully extended CERs. It predicts a single-gel phase rather than separated phases. The special feature of the model, however, is based on the liquid-like layer observed in the lamellar mid-plane observed in several LM model systems [13]. This layer is a result of the longer $>$ C20 CER chains and fatty acids which are by far the major proportion found within the LM [49]. These longer chains are overlapping in the lamellar mid-plane. Since they are less densely packed and have more freedom for lateral movement in this region, they behave more like oil or liquid than the rigid outer regions [13] (Fig. 4).

The liquid-like middle layer could provide additional mechanical stability protecting against shear stress and compression, by allowing the more rigid outer layers to laterally or perpendicularly move against each other without introducing major defects. The different miscibility/ solubility properties of the 3 layers can also explain how the barrier functions and why it does it so effectively. The entropy versus enthalpy concept going hand in hand with this model assumes competing entropic and enthalpic forces. The entropy aims towards a maximum disorder driving any foreign molecule to distribute as uniformly as possible and moving into and through the LM to do so, driven by a local concentration gradient. The molecular interactions like the strong and weak non-covalent binding forces and the tight structures within the rigid outer regions, as enthalpic forces, however, hinder the free movement and uniform distribution. The better mechan-

State of the Art in Stratum Corneum Research: Part II ical miscibility and chemical solubility within the middle layer would lead to the trapping of foreign substances within it since the entropic force is not enough to overcome the energy gradient, resulting from the enthalpic force in the form of different miscibility and solubility properties (a more detailed explanation of this concept is given in part I) [13].

\section{New Insight into the LM Properties}

In part I of this review series, the general biophysical properties of CERs were assessed and discussed. This part will, thus, focus on how these and other findings influence or may change our view on the lamellar micro- and molecular nanostructure of the LM. It will be highlighted in which way the hypothetical models introduced above are still holding up, considering these new findings. So far, there is no real consensus concerning the different models trying to describe the SC LM architecture and all of them are controversially discussed. It is certainly clear, however, that not all models are in accordance with each other. However, as discussed in part I of this review, it is clear that different CERs sometimes show an almost fundamentally different influence on the behaviour of the SC lipid phase even though chemical differences may be very minor. These differences can at least partially explain the different experimental observations that led to the differing models. Studies often used single CER components with different head groups or chain length. So far, there is no uniform definition for the categorization/nomenclature of LM lipids by chain length. Sassa et al. [66] recently suggested a system to categorize fatty acids by chain length, which can be adapted also for other lipids like CERs.

The studies that used mixtures of isolated CERs from natural sources furthermore partially utilized lipids with 


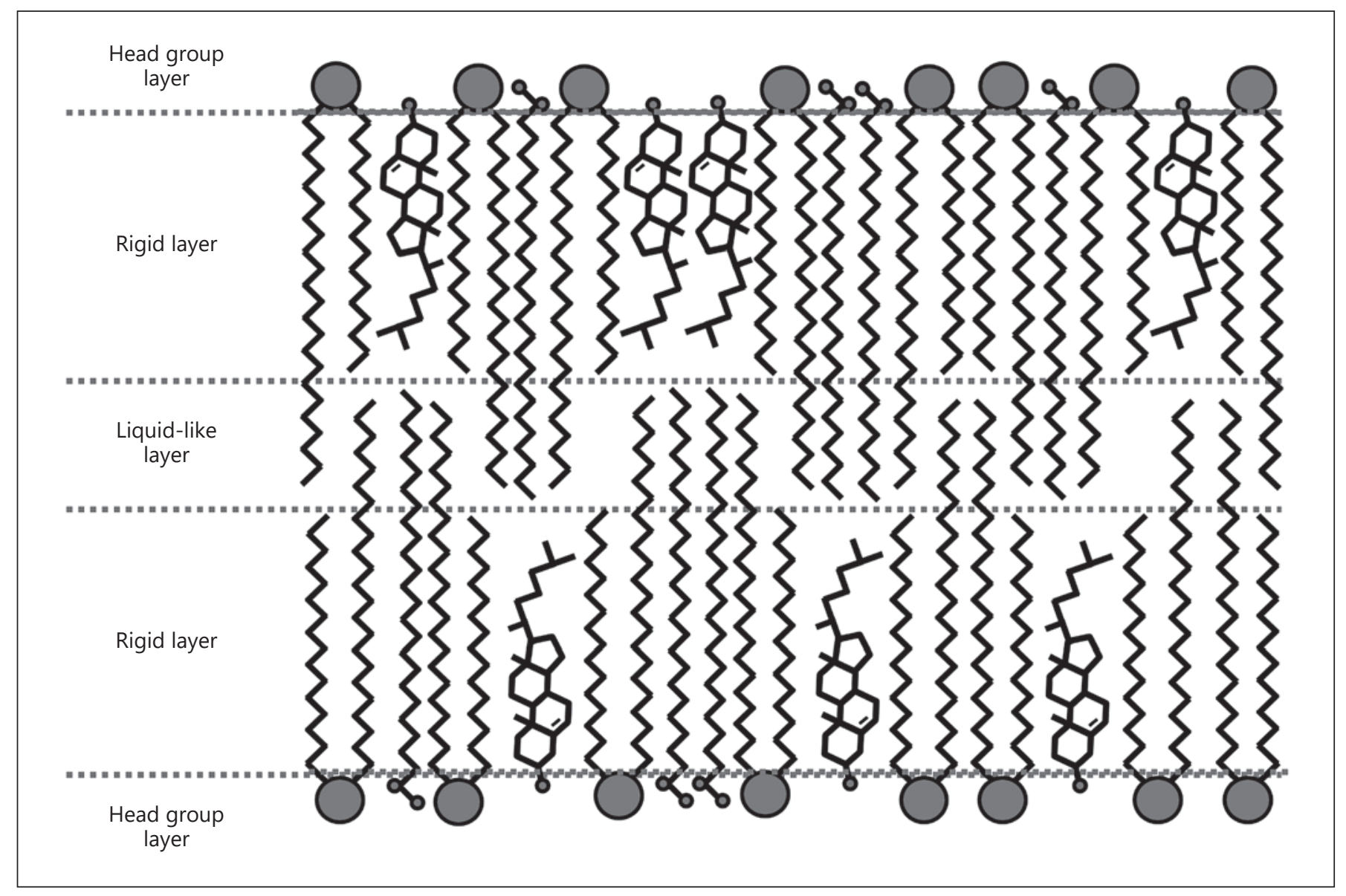

Fig. 4. Schematic representation of the LM architecture according to the tri-layer model. LM, lipid matrix [13].

different head groups or chain lengths and mixed them in different ratios. These studies also mostly used isolated pig CERs, which are known to have a different composition than the CERs in human SC [52]. These different experimental conditions make it harder to extract which observations are relevant for the native LM and which are mainly caused by different experimental conditions. The most useful experiments for understanding the basic underlying principles of the SC LM at this point are, thus, the mixtures that use single or very few well-defined CER components. These experiments allow for a better assessment of the general biophysical properties of single CER species. This way it is possible to decipher the properties of the SC LM step by step identifying the function of each individual lipid component of the SC as well as the basic interactions between them. Knowing the basic underlying molecular interactions, it will also be much easier to interpret results found for more complex systems or the native SC as demonstrated in part I [13]. Results for single lipids are further more useful to perform molecular simulations, which can then also be gradually built up to represent more complex compositions.

\section{Lateral and Phase Organization}

The lateral organization of the SC LM lamellae is a highly discussed topic. The 2 models that disagree the most in this aspect are the domain mosaic and the singlegel phase model. The first one assumes a separation between differently packed and composed domains. On the other hand, the latter proposes no phase separation within the SC LM. One factor that can influence miscibility for long aliphatic components such as lipids is the hydrophobic chain matching enhancing the hydrophobic interactions and optimizing the space requirements. In a study using ${ }^{2} \mathrm{H}$ nuclear magnetic resonance with a CER[NS] $\mathrm{C} 16, \mathrm{CHOL}$, and palmitic acid (PA) mixture, the influence of the hydrophobic chain matching was investigated. The study reported strong separation of the compo- 
nents despite the matching chains[67]. The separation of shorter chain CER[NS] is in contrast to results reported for CER[NS] isolated from porcine skin, with mixed chain length, for which the miscibility was much better [68]. The hydrophobic matching has, thus, a lesser influence on miscibility than the chain length distribution. The same was shown comparing CER[NS] C16 and C24 mixed with either lignoceric acid (LA) or a mixture of FFAs, CHOL, and with or without $\mathrm{ChS}$ at different humidities and temperatures using Fourier-transformed infrared spectroscopy (FTIR) [59]. Another FTIR study investigated the mixing properties of CER[NS] C18 and C24 in comparison, together with FFAs which were either PA, LA, or a mixture of FFAs. All of the mixtures formed semi-stable mixed crystals. The longer LA and the FFAs mix led to quite stable crystals that did not phase-separate during a time span of 3 months and showed only minimal phase separation under hydration. The PA on the other hand led to a strong phase separation which was even stronger under hydration [69]. The reported low miscibility of symmetric short-chain CER[NS] C16 as well as C18 is in agreement with previous studies, based on comparable mixtures $[67,70]$. It is mostly a result of the high rigidity and strong intramolecular interaction of the 2 lipid chains in the region where they are in direct proximity which results in weaker interactions with other molecules. Recently, a molecular simulation study also found that the double bond specifically disturbs the bilayer packing, resulting in a lower compressibility modulus and lager area per lipid [71]. These results demonstrate that the stiffer double bond forces the sphingosine CER into a conformation which is unfavourable for their mixing behaviour. This can partially explain findings showing a phase separation in several older experiments, mostly using CER[NS]. This may have led to the development of the domain mosaic model. However, most of the more recent studies report no phase separation. Also for mixtures of CERs with a more complex composition, closer to the natural LM, usually no phase separation is observed $[46,72]$. This was even shown for less complex mixtures, of one to 3 CERs using FTIR. In one study, for example, it was demonstrated that especially CER[NP] C18 alone showed a strong head group interaction, but also phase separation, even after phase transition. The more the CERs contained in the system, the more stable the mixing behaviour was, especially the 3 lipid systems containing also CER[AP] and [NS] C18 showed a stable mixing with a one-step phase transition at a comparably low temperature [73]. Phase separation was furthermore also shown to be temperature and chain length dependent in a mix-

State of the Art in Stratum Corneum Research: Part II ture with a more natural-like lipid composition which suggests no phase separation for the native LM at the skin temperature $\left(32^{\circ} \mathrm{C}\right)[72]$.

The domain mosaic model further implies the need for a minimal exchange of water and other substances through the SC [60]. This exchange could presumably be ensured due to the more permeable liquid domains. However, the desquamation cycle is a mechanism of complete molecular renewal of the SC every few days [74-76]. Furthermore, the directed and well-controlled excretion of substances via the skin is realized by the appendices of the skin, the sweat as well as the sebaceous glands. This basically negates the need for any further molecular exchange via the SC. It was also demonstrated that a more liquid phase behaviour, for example, observed in Netherton syndrome patients, caused by the accumulation of unsaturated FFAs in the skin, substantially impairs the barrier function of the LM [57, 77]. A real separation into actual physical domains is thus unlikely, and considering the current state of the art, most facts appear to be contradicting the domain mosaic model. It cannot be definitely concluded that absolutely no phase separation takes place but at least a liquid lateral phase is not to be expected. In model systems, usually a dense orthorhombic chain packing is observed which changes to a less dense hexagonal packing under hydration at $32^{\circ} \mathrm{C}[78-$ 80]. Some simple model mixtures show slightly different properties, but packings are always either predominantly hexagonal or orthorhombic. In full thickness skin samples, using attenuated total reflectance-FTIR, it could be shown that human and pig CERs have a different natural chain packing. Porcine skin features a predominantly hexagonal and human skin a predominantly orthorhombic packing [52]. It was shown that the change from an orthorhombic to a hexagonal chain packing had no notable effect on the barrier function [77, 81]. Comparing mixtures of CER[EOS] together with either CER[NS], $[\mathrm{AS}],[\mathrm{AP}]$, or $[\mathrm{NP}]$ together with $\mathrm{CHOL}$ and a mixture of FFAs, with a CER/CHOL/FFA ratio of 1:1:1, it was observed that the CER[AP] led to the lowest density chain packing of the mixtures. However, despite this, the mixture showed a very low permeability, which was contributed to the strong head group hydrogen bonding, demonstrating that a slightly lower chain packing alone does not necessarily impair the barrier function [82]. A different study, investigating the degree of orthorhombic versus lower density packing throughout several SC layers on the other hand found a slight correlation towards a higher barrier proficiency in individuals with an orthorhombic packing throughout all investigated skin layers 




Fig. 5. Schematic hypothetical representations of nanodomains found within the single-gel phase.

compared to the ones with a less densely packed LM [83]. However, the correlation is not very strong with a high variability between all the patients, it is thus not clear which other factors besides the number of more densely packed layers might have influenced the results. However, the chain packing definitely seems to be much less important for a proper barrier function than the homogenous lamellar organization [81] which will be discussed in a later section. One way to explain the differences in packing is the assumption of the presence of local nanocrystallites or low-density nanodomains within the otherwise continuous phase arrangement. The above described results demonstrate that even if the skin would have somewhat larger sized domains of differently packed lipids, it would hardly have a strong influence on the barrier function, as long as they are not in a liquid-like state. If nanocrystallites or low-density nanodomains as shown in Figure 5 would be present, just limited disturbance of the barrier function would to be expected.

The formation of nanoscopic crystallites can be assumed to result from the high rigidity of CERs and their low miscibility with other lipids. They would form by grouping together because of the stronger homologous interaction of CERs compared to that with other lipids or because of the grouping of long- or short-chain lipids in separate regions. This is basically the same mechanism as described for the formation of lipid rafts within the cellular membrane only without the participation of any lipophilic proteins which are not present in the LM [84, 85]. Due to the equimolar ratios of CERs, CHOL, and FFAs, the domain size would, however, be very limited. Using X-ray powder diffraction, it could be demonstrated that the CHOL had a very limited influence on the overall chain packing of mixtures of synthetic as well as isolated CERs. The CHOL-depleted systems did furthermore show even decreased permeability, suggesting a different role for the CHOL in the SC [86]. Utilizing the natural abundance of ${ }^{13} \mathrm{C}$, it could be shown that only a small proportion of the lipids actually show increased mobility, which slightly increased with hydration [87]. Using MDS, another study was focused on CER[NS] C16 in a mixture with $\mathrm{CHOL}$ and FFAs. It suggested a very important role of the CHOL, which leads to a higher lateral area per CERs and FFAs and thus a lower chain ordering which improves miscibility. It increases the compressibility and, thus, the mechanical stability of the LM and provides fluidity below and rigidity above the phase transition temperature and stabilizes the miscibility [88].

The observation that there is only a small proportion of mobile lipids could, thus, be explained by the properties of the CHOL. Due to its smaller head group, the $\mathrm{CHOL}$ is less tightly bound within the strong $\mathrm{H}$-bonding network of the SC lipid head groups and could, thus, be liberated from it by an exchange to a different $\mathrm{OH}$-group, for example, of a water molecule as interaction partner. The CHOL and possibly a limited number of neighbouring molecules would, thus, be fluidized, migrating more freely. This would also explain the increased mobility upon hydration. Despite the low-chain mobility of CERs on a molecular scale, there are still dynamic processes within a nanosecond timeframe preventing the formation of larger domains. This includes especially the described effect of the CHOL. The necessity of a minimum ratio of about $9 \mathrm{~mol} \%$ for the formation of a uniform SPP without phase separation was shown before highlighting the importance of CHOL for the lipid miscibility [46]. 
The above described processes, mainly the fluidizing effect of the CHOL, would lead to a constant resolution and reformation of any nanoscopic domains, upholding an overall gel- or crystalline-like packing and limiting the domain size as well as retention time. It has furthermore to be considered that the tri-layer model assumes a change in lipid mobility = fluidity/liquidity along the perpendicular axis which could possibly also explain the observation of different phases [13], which will be discussed in the upcoming section. As mentioned above, it has to be further considered that according to the sandwich model, the liquid phase proportion could be attributed to a perpendicular layer of the LPP [26-28], which will not be discussed in further detail since this review is focusing on the SPP.

Another MDS study furthermore found that CHOL within the SC seems to have the possibility to perform a fast flip-flop movement changing between lamellar leaflets. This could be of importance for the adaption to SC curvature which is constantly changing in some SC regions [89]. The mechanical flexibility could furthermore be aided by the 2 effects of the above-mentioned perpendicular liquid layers [13, 26-28], which will be discussed in further detail in the upcoming section. A liquid lateral phase proportion would, thus, also not be necessary for the mechanical stability or adaptability of the LM to mechanical influences, making such an arrangement even less likely. Considering all these results as far as a concept for the lateral phase arrangement goes, the single-gel phase model seems by far the more likely concept than the domain mosaic model.

\section{Perpendicular Lamellar Layer Microstructure}

The microstructural arrangement of the SC lipids into lamellar layers is a highly discussed topic as well. The first important aspect of the microstructure is the lamellar thickness. For model systems solely based on short-chain CERs, shorter 4-4.5-nm and for >C20 CERs 5-6.5-nmthick lamellae as usually described for the natural SPP are observed. In the native LM, both shorter and longer bilayer units are found $[29,54,58]$. However, the longer CERs seem to have a special purpose which cannot be compensated by the shorter CERs. Already, a slightly increased ratio of short-chain CERs was observed for atopic eczema. However, a slightly decreased amount of longchain CERs can disturb the barrier function [90]. This is hinting to a special purpose of the longer lamellar arrangement. It was observed that the lamellar thickness for the longer arrangement is usually a bit less than for $2 \mathrm{di}$ rectly opposing long chains due to overlapping in the la-

State of the Art in Stratum Corneum Research: Part II mellar mid-plane [88, 91-95]. Details about this feature will be discussed later. The lamellar thickness also decreases proportional to the lateral phase ordering [96]. This is an effect of the increased lateral space availability and thus lateral movement. In response, the perpendicular space requirement is most likely reduced. This effect is, however, rather small, only changing the thickness by about $1 \mathrm{~nm}$ [12]. Since the lateral ordering is most likely a mostly continuous single-phase arrangement with only semi-stable nano-sized domains, this effect would have a constant negligible contribution to the overall lamellar thickness. The armature reinforcement model suggests a change in lamellar thickness or swelling upon hydration of the lipid lamellae. This effect is proposedly caused by a chain conformation change from the fully extended to the hairpin conformation. Because this fact reduces the head group cross-linking caused by $\mathrm{H}$-bonds or the armature reinforcement, the spacing supposedly increases by water uptake into the interlamellar space [64]. However, this is not in agreement with the general observation of few or no bound water molecules also leading to only a neglectable swelling of the lipid lamellae caused by hydration $[89,97,98]$. A swelling of native skin was shown to be a result of cellular swelling due to a water uptake hydrating the intracellular proteins and not by a change in lamellar structure [17]. Some studies also observe either a Vshaped or a tilted arrangement of the CERs which is influencing the lamellar thickness [56, 92, 99-102]. While a small angle between the chains and/or a small proportion of V-shaped CERs would certainly be possible $[56,102]$, an arrangement with mostly strongly V-shaped CERs would hardly be possible within the native LM. It would lead to gaps or less dense areas in regions where lamellae are arranged in different directions laterally connect, which would negatively influence the barrier function. Because all of these factors are expected to be static under physiological conditions, the lamellar thickness can also be expected to be fairly constant. The only expectable dynamic influence would be a small increase of lamellar thickness with increasing temperature due to the increased molecular movement and thus area requirement per lipid. This would, however, also be a very minimal effect within a physiological temperature range [103, 104].

As a result of the regional difference in thickness a macroscopic lamellar layering can be observed. Some studies find an ordered macroscopic layering with repeating units giving a specific band pattern in the electron micrographs [58, 105]. Other results describe a mostly random layering [57]. The ordered layering was attrib- 
uted primarily to the influence of the very long $\omega-\mathrm{OH}$ CERs and thus rather to the LPP than to any SPP structure [26-28]. The asymmetry (splayed) bilayer model, however, proposes the possibility of a macroscopic ordering even without the influence of the very long $\omega-\mathrm{OH}$ CERs [58]. Important for such an ordered lamellar layering independent of the very long $\omega$-OH CERs would be some kind of other structural influence. According to the asymmetry bilayer model, this could be a result of the fully extended conformation of the 2 CER chains. With the long and short chains exclusively arranged in different sub-cells, it results in long and short lamellae like in the model originally proposed by Iwai et al. [58]. This model would explain the $4-4.5-\mathrm{nm}, 5-6.5-\mathrm{nm}$, and $\geq 11-\mathrm{nm}$ (LPP) arrangements observed in the natural SC with only 1 type of lamellar arrangement with a combined $6.5+4.5$ $\mathrm{nm}(11 \mathrm{~nm})$. It was furthermore reported that CER[NH] which is currently hardly investigated favours the formation of similar 10-11-nm repeating units $[106,107]$.

This is somewhat in contrast to most structural studies using a diffraction method for the investigation of the lamellar structure of the SC lipids. Basically, all diffraction studies using model systems so far concluded a symmetric hairpin conformation since it fits the observed results best in most cases $[95,108,109]$. However, the difference in length between fully extended and hairpin-folded CERs would be minimal and could easily be mistaken for a small chain tilt, a result of chain disordering or simply a measurement error if the effect is even prominent enough to be detectable at all if the resolution is high enough. In fact, the small difference in length between the CER[NP] and [AP] which was observed in 2 neutron diffraction studies could indicate a different head group interaction shortening the chain somehow as discussed in the original work [31, 92]. However, it could also hint towards a full extended conformation for the CER[AP]. Just like in these studies, so far always only one of the 2 CER chains could be visualized by deuterium labelling. This makes it harder to get any definitive result for their chain conformation. For the model systems, it has furthermore to be considered that not necessarily all layers are arranged in the same direction. This may yield misleading results and possibly be falsely indicating a symmetric distribution due to several differently oriented asymmetric layers mirroring each other. The preparation of the model system is furthermore severely lacking, considering physiological lamellae formation. First of all, there is no anchor or scaffold as the LE provides in the native SC [5-7]. This could already change the resulting structure of the model system which is in this case solely resulting from a thermally induced self-assembly. This process is very different from the process of step-by-step deposition of preassembled lamellae from the lamellar granules, adding more uncertainty about the structure formation. Naturally, the LM is formed by the extrusion of pre-packed lamellae from the lamellar granules into the extracellular space which then only unfold. While model systems are equilibrated by spraying and thermal treatment, the natural lipid lamellae may not necessarily be in equilibrium. They possibly yield an only semi-stable arrangement upheld by the limited degree of molecular dynamics within the relatively rigid LM.

However, it has to be taken into account that in the CER lamellae, even though they are very rigid, there are still dynamic processes on a nanosecond timescale. These allow for at least some degree of reassembly of the structure and the formation of an arrangement favoured by the given lipid interactions [87-89, 110, 111]. Particularly, the CHOL and shorter CER or FFA chains could change plane and, thus, easily disrupt any strictly ordered arrangement. Especially, the CHOL was described to be able to perform a fast flip-flop mechanism enabling it to do so [89]. This makes the existence of a semi-stable arrangement as predominant structure less likely. Particularly, the solely asymmetric distribution of CHOL being only associated with the short chains appears to be unlikely since it was never reported to be completely immiscible with longer FFA or CER chains, thus at best an enrichment of CHOL within the short 4.5-nm layer would be possible. However, the rearrangement mostly concerns the CHOL as well as the lipid chains only [89]. In contrast to that, the head groups are mostly statically bound within the $\mathrm{H}$-bonding network because of that the degree of reassembly is still relatively limited. Most important is also the tight lateral and perpendicular packing within the natural LM, without bordering any fluid layer. The additional templating by the LE should contribute highly to stabilizing the lamellar structure. This result is in contrast to the situation produced in simulation studies where mostly 1 or 2 lamellae are studied bordered by water layers.

Some MDS even suggest the tendency for the CERs to rather form inverse micellar instead of lamellar structures over time without any templating [111, 112]. A strongly oriented arrangement of the SC lipids would, thus, also need some kind of internal perpendicular templating. Since there are no proteins or other macroscopic structures in the region of the SC lipids which could lead to such an effect, the only possible influence could be originating from CERs themselves. One possibility would be 
the long- $\omega$-OH CERs. These were already shown to form a phase arrangement different from the described SPP and LPP, which will not be discussed in detail here. However, particularly saturated variants were also integrated into the SPP, possibly stabilizing its structure $[31,113]$. However, the low amount of $<10 \%$ long $\omega$-OH CERs [49] would hardly be sufficient to explain such an ordered arrangement over the whole thickness of the LM as the splayed bilayer model describes. The splayed bilayer model suggests the better miscibility of lipid chains with different length as possible templating influence leading to the splayed bilayers with fully extended CERs arranged asymmetrically [58]. This is true for the lateral miscibility [114] and could, thus, explain the asymmetry of the monolayer unit. However, the same is not to be expected for the perpendicular association of the chains, with only the terminal carbons in direct contact. These contradictions would leave the repeating broad-narrow bilayer unit and, thus, the macroscopic layering unexplained. However, there is one more possibility to be considered which could favour an asymmetric layer distribution. Instead of increased attraction, the main influence on the separation in broad and narrow layers could be increased repulsion of the chain ends. The cause would be the large inequality in chain length between the long (mainly C22-C34) fatty acid chains and the short (C16-C18) sphingoid base chains $[49,115]$. This effect would lead to a more or less sharp tail end region for the short and a very irregular one for the long chains. End-to-end connections between equal lamellar monolayer units with substantial chain overlapping in the mid-plane for the long chains would, thus, be favoured. Particularly, the properties of the shorter C16-18 CERs would further contribute to this effect. The symmetric short-chain CERs were already shown to not mix well with other lipids. It was suggested also that short-chain CERs seem to prefer a hairpin folding, while long-chain CERs favour an extended conformation [59, 116]. This effect would also explain the "island of stability" leading to an enrichment in particularly the symmetric $\mathrm{C} 16$ and $\mathrm{C} 18$ CERs and the following gap to the main CER proportion starting at about C22 $[49,115]$. The main argument against such an arrangement would be a general lack of short-chain CERs $[49,115]$ and FFAs [117]. There would be too little short-chain CERs to lead to such a frequent repeating of the shorter arrangement as observed within the electron micrographs $[55,56]$. However, it was observed that the short lipids also mix better with $\mathrm{CHOL}$ which has about the same perpendicular space requirements. Because it represents about $1 / 3$ of the overall lipid molecules, the suggested enrichment of this

State of the Art in Stratum Corneum Research: Part II lipid in the short layers could, thus, easily compensate for this lack in short CERs and FFAs. This phase behaviour could yield exactly the same pattern as described for the asymmetry bilayer model[55-58] but without necessarily featuring only fully extended CERs and CHOL only in the short layer. This arrangement would, however, lead to different layer properties because as discussed above, the short-chain CERs behave different from the long-chain CERs, and the head group region would consist of 2 opposing head groups instead of one extended head group with less cross-linking between the 2 leaflets. This would mainly change the fluidity behaviour of the lamellae and make them more flexible.

The observed tendency for at least some CERs to form a fully extended conformation makes it very likely that at least a certain proportion of CERs yields a fully extended conformation within the SC $[59,116]$; however, no real estimate on the proportion can be given at the moment. Considering that the CERs show sometimes fundamentally different molecular interactions as discussed in part I [13], it is unlikely that all of them will favour the same conformation. For the phytosphingosine-based CERs, for example, a stronger in-plane $\mathrm{H}$-bonding was observed than for the sphingosine CERs [118]. For the CER[NS] in contrast to this, a tendency for perpendicular H-bonding with the adjacent head group region of the next layer was reported. Despite the fact that both are sphingosine-based CERs, this effect was not reported for the CER[AS] [119, 120]. Perpendicular H-bonding between 2 different layers would hardly be possible for fully extended CERs, while stronger in-plane $\mathrm{H}$-bonding could suggest both depending on the plane. There could also be dynamic changes between fully extended and hairpin conformation as suggested by the armature reinforcement model, with different CERs having a different equilibrium ratio of fully extended and hairpin-folded CERs under different environmental conditions. This could, for example, buffer different physical influences as was suggested also for other differences between the CER species in part I of this review series [13].

One bilayer would consequently be containing the short base chains of a certain proportion of fully extended long CERs together and most short-chain CERs, short FFAs and CHOL as suggested for the splayed bilayer model [55-58]. This layer would be paired with a bilayer of the long CER chains, long FFAs, and a certain proportion of hairpin-folded long-chain CERs as well as a smaller proportion of CHOL, short CERs, and short FFAs. It can, thus, be concluded that the asymmetry (splayed) bilayer model [55-58] as well as the tri-layer model [13] 
most likely describe the structure of natural LM reasonably well, agreeing in most aspects. The proportions of fully extended and hairpin-folded CERs are not certain at the current state of the art and would be dependent on the individual CER properties. Because of the different phase properties of short and long CERs, such an arrangement would allow for a self-assembly leading to the broad and narrow repeating pattern with a combined $\sim 11-\mathrm{nm}$ repeating unit as described by the splayed bilayer model [55-58] or with longer CERs, possibly also longer (12-14 $\mathrm{nm}$ ) units. The main argument against this hypothesis is so far the lack of experimental proof of some of these aspects. As mentioned above, no asymmetric arrangements outside of the native skin or the LPP were observed, not even for model systems containing both short- and longchain CERs. Consequently, there is hardly any experimental proof supporting any of these 3 possibilities so far. This could be a problem of the experimental design as discussed above.

\section{Perpendicular Molecular Nanostructure}

As described in part I of this review series, CERs have some general features which seem to be very important for the formation of a specific molecular nanostructure and consequently the function of the skin barrier. The first of these features are the small head groups which solely interact via strong directed $\mathrm{H}$-bonds. To penetrate the head group layer, first several $\mathrm{H}$-bonds would have to be broken which would require a lot of energy. Even the water is very tightly bound within the network, forming a rigid net-like structure preventing the penetration of large, bulky, and nonpolar molecules through this layer but also limiting the interaction of polar substances with the head groups of the SC lipids as described in part I [13].

The next important feature is the large chain length of the CERs of mainly C24-C34 [49, 115]. As discussed above, even the enrichment in short symmetric CERs can cause barrier disturbances [90]. Very short-chain CERs, even if applied to the skin, strongly disrupt the skin barrier by disturbing the head group interactions and chain ordering $[118,121-123]$. The most important new result regarding the long chains, however, is the chain interdigitation in the lamellar mid-plane due to the chain asymmetry which was shortly mentioned above. In several studies, an arrangement with the long CER and FFA chains overlapping in the lamellar mid-plane resulting in a less tightly packed arrangement of the SC lipids was reported [88, 91-95]. The reduced chain density and order would most likely lead to more liquid-like behaviour compared to the very rigid outer regions. As discussed for the entropy versus enthalpy principle in part I of this review series, the perpendicular layer arrangement according to the tri-layer arrangement could very well be the key to understanding the function and effectiveness of the skin barrier. As described for the tri-layer model, both the hairpin conformation and the fully extended conformation or mixed proportions of both as the armature reinforcement model proposes would be a possibility. The differences in chain length would lead to the overlapping layer in either case [13]. Even if all the long chains would be arranged in 1 direction and opposed by other long chains as suggested by the splayed bilayer mo,el [58] due to the difference in length of the different FFAs and other lipids, sharp ends of the bilayer sub-cells would, thus, not be possible. Considering the high energy cost and negative effect of large gaps on the barrier function, an overlapping middle layer would inevitably be the result as discussed by Lundborg et al. $[55,56]$ with the effect being clearly visible in the supplementary video as well. Even for the shorter bilayer mainly containing symmetric C16C20 CERs, shorter FFAs, and CHOL, a certain degree of overlapping is to be expected even though it should be much less because the differences in chain length are much smaller. The smaller overlapping region could be even more stable against shear stress since only small chain overhangs wold have to slide past each other. The larger overlapping region could buffer compression from skin curvature, mechanical indentations, or swelling of the underlying cells upon hydration much better.

\section{Conclusion: The Unified Model of the SPP}

Scrutinizing all of the different models and comparing them to each other showed that most of them actually largely agree on certain important features of the LM. The models mainly focus on different observations highlighting different aspects of the organization. Taking all the factors, the models do agree on and factoring in the most recent experimental observation slightly updating some of them a model considering the state of the art concerning the SC LM at the moment can be suggested. This model is unifying all of the current models into 1, leaving some currently unknown details open for further inspection but without causing contradictions. The lateral chain organization would most likely be a single dense orthorhombic or slightly less dens hexagonal phase possibly varying between species. No huge drawback would be expected from the slightly less dense arrangement. Within these single-phase nanocrystallites formed by denser 


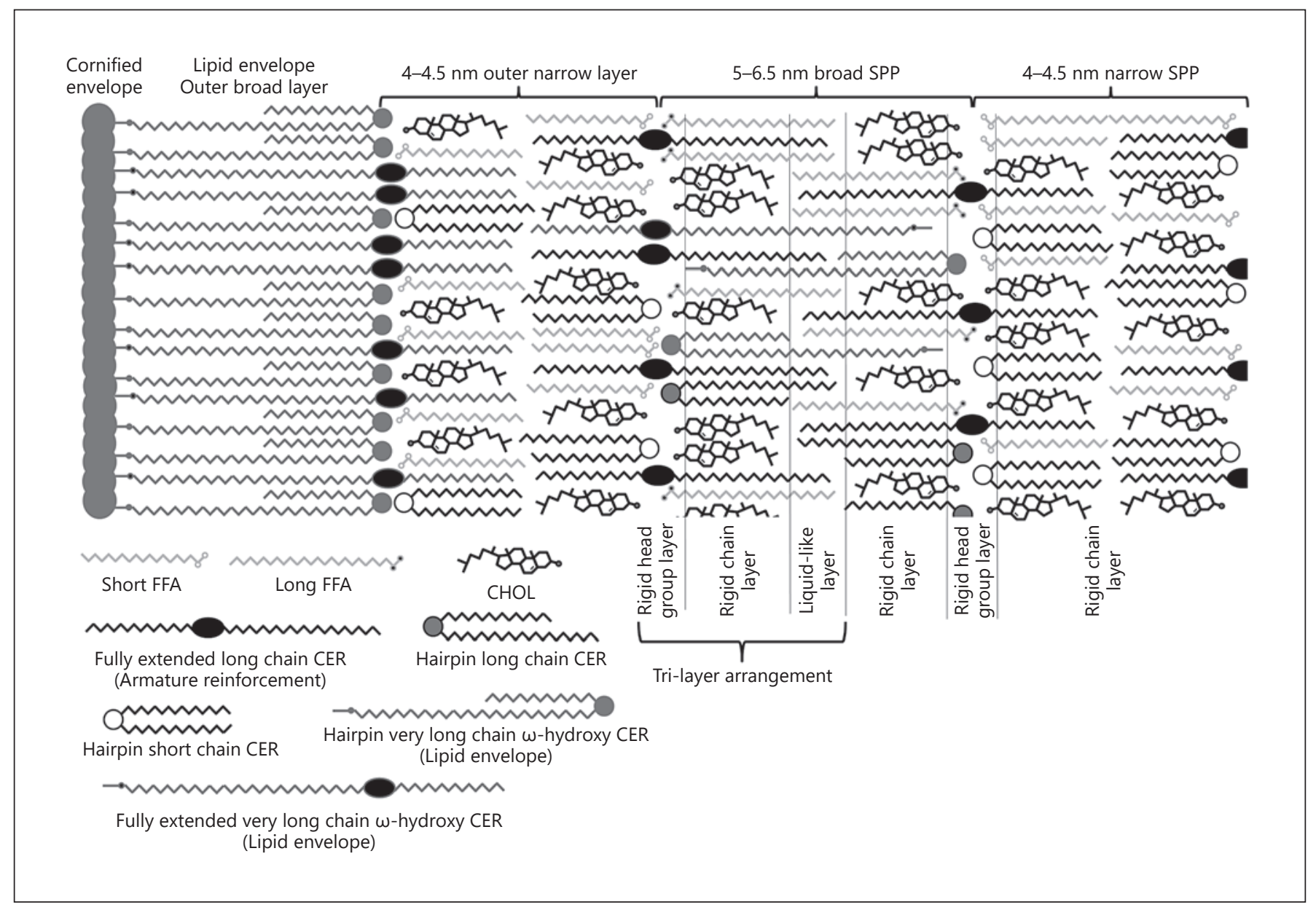

Fig. 6. The unified model of the native SPP (exact proportions of fully extended and hairpin-folded CER are unknown, no broad-broad arrangements shown). SPP, short periodicity phase; FFA, free fatty acid; CHOL, cholesterol; CER, ceramide.

clusters of CERs and/or FFAs and low-density nanodomains, the fluidizing effect of CHOL as shown in Figure 5 could be present. These nanodomains would dynamically resolve and reform, giving the lipid lamellae a certain degree of dynamics allowing for the adaption to skin curvature, mechanical damage, and more.

The macroscopic layer arrangement would externally be templated by the LE and internally by the CER properties. It is likely that lipids having short symmetric chains get concentrated in shorter 4-4.5-nm lamellae arrangements to some degree. These would most likely also contain most of the short chains of fully extended CERs. The long lipids/chains would get concentrated in 5-6-nm lamellae, forming a certain degree of broad-narrow repeating units. The presence of isolated $4-4.5$ or $5-6.5-\mathrm{nm}$ repeating units is unlikely but cannot definitely be excluded at the moment. Most likely $f$ the whole LM would feature

State of the Art in Stratum Corneum Research: Part II the $\sim 11$-nm broad-narrow pattern as described by the asymmetry (splayed) bilayer model.

No final conclusion about the chain conformation can be drawn yet. However, this review considered the differences between the properties of long asymmetric and short symmetric CERs as well as between CERs with different head groups. Cumulating all these factors makes it most likely that there is an equilibrium rather than an absolute state for the ratio between hairpin and fully extended CERs. Some CERs would mostly favour the fully extended. Some other CERs would preferably yield the hairpin conformation. However, it is not possible to make any estimation about the equilibrium proportions as of yet.

Despite which conformation is predominant, an overlapping middle layer as a result of the varying length of the fatty acid chains would always be present. The sug- 
Table 1. Categorization of LM lipids by lengths

\begin{tabular}{lcl}
\hline Categorization & Chain length & Properties \\
\hline Ultra/very short chain & $<16 \mathrm{C}$ & Disturb lamellar structure [58] \\
Short chain & $16-20 \mathrm{C}$ & About symmetric chain lengths \\
Long chain & $21-32 \mathrm{C}$ & Asymmetric chain lengths \\
Ultra/very long chain & $>32 \mathrm{C}$ & As long as/longer than SPP bilayer \\
\hline
\end{tabular}

LM, lipid matrix; SPP, short periodicity phase.

Table 2. Summary of features of the different theoretical SC LM models

\begin{tabular}{|c|c|c|c|c|c|c|c|c|}
\hline Model & Type & Methodology & Lipid source & Repeating unit & $\begin{array}{l}\text { Chain } \\
\text { packing }\end{array}$ & $\begin{array}{l}\text { Per- } \\
\text { meability }\end{array}$ & Layers & Peculiarities \\
\hline Brick and mortar & Basic & $\mathrm{eM}$ and $\mathrm{FF}$ & hSkin/pSkin & na & na & LM & na & COR impermeable \\
\hline Asymmetry & Molecular & $\begin{array}{l}\text { eM, XRD, } \\
\text { IRS, and MDS }\end{array}$ & hSkin/Syn & $4-6.5 \mathrm{~nm}$ & Ort/Hex & - & $\begin{array}{l}\text { Asymetric } \\
\text { bilayers }\end{array}$ & Asymmetric \\
\hline Domain mosaic & Microscopic & $\mathrm{XRD}$ & pSkin/Syn & na & Ort/Hex/Liq & Liq phase & Bilayers & Permeable liq phase \\
\hline Sandwich & Molecular & $\begin{array}{l}\text { eM, XRD, } \\
\text { and ND }\end{array}$ & hSkin/pSkin/Syn & $12-13 \mathrm{~nm}$ & Ort/Hex & - & Triple layer & Liquid middle section \\
\hline Single-gel phase & Microscopic & $\begin{array}{l}\text { eM, XRD, } \\
\text { ND, and MDS }\end{array}$ & hSkin/pSkin/Syn & na & Ort/Hex & - & Bilayers & None \\
\hline $\begin{array}{l}\text { Armature } \\
\text { reinforcement }\end{array}$ & Molecular & ND & Syn & Var. $3-4.55 \mathrm{~nm}$ & Ort/Hex & Hydrated & Bilayers & $\begin{array}{l}\text { Swelling/ } \\
\text { conformation change }\end{array}$ \\
\hline Tri-layer & Molecular & ND and NMR & Syn & $5-6 \mathrm{~nm}$ & Ort/Hex & - & Bilayers & Fluid middle layer \\
\hline Unified & Molecular & Mixed & Mixed & $\begin{array}{l}4-4.5 \text { and } \\
5-6 \mathrm{~nm}\end{array}$ & Ort/Hex & - & $\begin{array}{l}\text { Mixed } \\
\text { bilayers }\end{array}$ & Mixed features \\
\hline
\end{tabular}

Bold face indicates different; roman type indicates similar; na indicates not determinable. COR, corneocyte; SC, stratum corneum; LM, lipid matrix; eM, electron microscopy; FF, freeze fracture microscopy; XRD, X-ray diffraction; IRS, infrared spectroscopy; MDS, molecular dynamics simulations; ND, neutron diffraction; NRM, nuclear magnetic resonance; pSkin, porcine skin; hSkin, human skin; Syn, synthetic lipids; Ort, orthorhombic; Hex, hexagonal; Liq, liquid.

gested more liquid-like properties of this layer could have an important effect on the stability against shear stress and compression and could be important for skin adaptability. Furthermore, this effect would be supported by the special properties of the CHOL, which has the highest mobility among the SC lipids and can even perform a flipflop mechanism between 2 opposing bilayers. According to the entropy versus enthalpy concept, the 3 layers as proposed in the tri-layer model are of utmost importance for the barrier function. The 2 rigid outer layers repel molecules and prevent penetration, and the middle layer could possibly have a trapping effect on penetrating molecules because it would have the best miscibility/solubility properties.

At this point, due to the lack of experimental data, some aspects of this model are still based on logical con- clusion rather than on experimental proof. As discussed above, it is still impossible, to draw any final conclusion on the chain conformation. So far, no actual penetration experiments with a nanometre resolution could be performed to actually show direct proof of the fluidity and diffusion behaviour throughout the lamellae. Because of the lack of methods to measure the structure of the LM in native skin, it is yet unclear what influence the CLE actually has on the structure and distribution of the LM lipids and can only be estimated. It has also to be taken into consideration that while CERs, FFAs, and CHOL are the main constituents of the LM, other lipids like cholesterol sulphate and traces of sphingolipids, phospholipids, triglycerides, and others can be found [124]. While these trace amounts most likely do not have a large impact on the lamellar structure and properties, 
they could, for example, serve the purpose of fine tuning the properties of the membranes. At last, the distribution of short- and long-chain CERs along the LM is a conclusion which is drawn from their mixing behaviour, molecular dynamic simulations, and the observed path lengths in models as well as the native SC, but no actual experimental proof of this feature in the native skin exists.

However, the model is able to explain most of the observed LM properties and geometrical as well as special features without causing any major contradictions. It is not contradicting any commonly agreed upon facts from other models and many of its features are, thus, still based on experimental prove and are generally accepted. A graphic representation of the unified model is presented in Figure 6. A summary of features of all models and if these fit the cumulated data which were considered for the unified model can be found in Tables 1 and 2 .

\section{Acknowledgements}

The study was supported by the Deutsche Forschungsgemeinschaft (DFG NE 427/30-1, and DO 463/6-1) and by Evonik Industries AG (stipend for T. Schmitt). We especially thank Prof. Dr. Lars Norlén from the Department of Cell and Molecular Biology (CMB), Karolinska Institute, Stockholm, Sweden, and Dr. Magnus Lundborg from the ERCO Pharma AB, Science for Life Laboratory, Solna, Sweden, who helped develop and advance some of the discussed models themselves for pre-reviewing our manuscript and providing a valuable point of view.

\section{Conflict of Interest Statement}

The authors declare no conflict of interest.

\section{Funding Sources}

The authors did not receive any funding.

\section{References}

1 Tregear RT. Relative penetrability of hair follicles and epidermis. J Physiol. 1961;156:30713.

2 Scheuplein RJ. Mechanism of percutaneous adsorption. I. Routes of penetration and the influence of solubility. J Invest Dermatol. 1965;45:334-46.

3 Kitajima Y. Regulation and impairments of dynamic desmosome and corneodesmosome remodeling. Eur J Dermatol. 2013. Online ahead of print.

4 Wertz PW, van den Bergh B. The physical, chemical and functional properties of lipids in the skin and other biological barriers. Chem Phys Lipids. 1998;91:85-96.

5 Behne MJ, Uchida Y, Seki T, Montellano PO de, Elias PM, Holleran WM. Omega-hydroxyceramides are required for corneocyte lipid envelope (CLE) formation and normal epidermal permeability barrier function. J Invest Dermatol. 2000;114:185-92.

6 Elias PM, Gruber R, Crumrine D, Menon GK, Williams ML, Wakefield JS, et al. Formation and functions of the corneocyte lipid envelope (CLE). BBA. 2014;1841:314-8.

7 Stewart ME, Downing DT. The omega-hydroxyceramides of pig epidermis are attached to corneocytes solely through omega-hydroxyl groups. J Lipid Res. 2001;42:1105-10.

8 Weerheim AM, Ponec M. Determination of stratum corneum lipid profile by tape stripping in combination with high-performance thin-ayer chromatography. Arch Dermatol Res. 2001;293:191-9.

9 Scheuplein RJ. Permeability of the skin: a review of major concepts and some new developments. J Invest Dermatol. 1976;67:672-6.
10 Rerek ME, van Wyck D, Mendelsohn R, Moore DJ. FTIR spectroscopic studies of lipid dynamics in phytosphingosine ceramide models of the stratum corneum lipid matrix. Chem Phys Lipids. 2005;134:51-8.

11 Schroeter A, Kiselev MA, Hauss T, Dante S, Neubert RHH. Evidence of free fatty acid interdigitation in stratum corneum model membranes based on ceramide [AP] by deuterium labelling. BBA. 2009; 1788:2194-203.

12 Ruettinger A, Kiselev MA, Hauss T, Dante S, Balagurov AM, Neubert RHH. Fatty acid interdigitation in stratum corneum model membranes: a neutron diffraction study. Eur Biophys J. 2008;37:759-71.

13 Schmitt T, Neubert RHH. State of the art in Stratum Corneum research: The biophysical properties of ceramides. Chem Phys Lipids. 2018;216:91-103.

14 Michaels AS, Chandrasekaran SK, Shaw JE. Drug permeation through human skin: theory and invitro experimental measurement. AIChE J. 1975;21:985-96.

15 Elias PM. Epidermal lipids, barrier function, and desquamation. J Invest Dermatol. 1983; 80:44-9.

16 Elias PM. Epidermal lipids, membranes, and keratinization. Int J Dermatol. 1981;20:1-19.

17 Bouwstra JA, Gooris GS, Vries MAS-D, van der Spek JA, Bras W. Structure of human stratum corneum as a function of temperature and hydration: a wide-angle $\mathrm{X}$-ray diffraction study. Int J Pharm. 1992;84:205-16.

18 Kessner D, Ruettinger A, Kiselev MA, Wartewig S, Neubert RHH. Properties of ceramides and their impact on the stratum corneum structure. Part 2: stratum corneum lipid model systems. Skin Pharmacol Physiol. 2008;21:58-74.
19 Breathnach AS, Goodman T, Stolinski C, Gross M. Freeze-fracture replication of cells of stratum corneum of human epidermis. J Anat. 1973;114:65-81.

20 Swartzendruber DC, Manganaro A, Madison KC, Kremer M, Wertz PW, Squier CA. Organization of the intercellular spaces of porcine epidermal and palatal stratum corneum: a quantitative study employing ruthenium tetroxide. Cell Tissue Res. 1995;279:271-6.

21 Madison KC, Swartzendruber DC, Wertz PW, Downing DT. Presence of intact intercellular lipid lamellae in the upper layers of the stratum corneum. J Invest Dermatol. 1987;88: 714-8.

22 Hou SY, Mitra AK, White SH, Menon GK, Ghadially R, Elias PM. Membrane structures in normal and essential fatty acid-deficient stratum corneum: characterization by ruthenium tetroxide staining and X-ray diffraction. J Invest Dermatol. 1991;96:215-23.

23 Downing DT. Lipid and protein structures in the permeability barrier of mammalian epidermis. J Lipid Res. 1992;33:301-13.

24 Fartasch M, Bassukas ID, Diepgen TL. Disturbed extruding mechanism of lamellar bodies in dry non-eczematous skin of atopics. Brit J Dermatol. 1992;127:221-7.

25 Swartzendruber DC. Studies of epidermal lipids using electron microscopy. Semin Dermatol. 1992;11:157-61.

26 Bouwstra JA, Gooris GS, Dubbelaar FE, Weerheim AM, Ponec M. pH, cholesterol sulfate, and fatty acids affect the stratum corneum lipid organization. J Investig Dermatol Symp Proc. 1998;3:69-74.
State of the Art in Stratum Corneum Research: Part II
Skin Pharmacol Physiol 2020;33:213-230 DOI: $10.1159 / 000509019$ 
27 Bouwstra JA, Gooris GS, Ponec M. The lipid organisation of the skin barrier: liquid and crystalline domains coexist in lamellar phases. J Biol Phys. 2002;28:211-23.

28 Bouwstra JA, Gooris GS, Dubbelaar FER, Ponec M. Phase behavior of lipid mixtures based on human ceramides: coexistence of crystalline and liquid phases. J Lipid Res. 2001;42:1759-70.

29 Al-Amoudi A, Dubochet J, Norlén L. Nanostructure of the epidermal extracellular space as observed by cryo-electron microscopy of vitreous sections of human skin. J Invest Dermatol. 2005;124:764-77.

30 Wilkes GL, Nguyen A-L, Wildnauer R. Structure-property relations of human and neonatal rat stratum corneum I. Thermal stability of the crystalline lipid structure as studied by $\mathrm{X}$ ray diffraction and differential thermal analysis. BBA GS. 1973;304:267-75.

31 Schmitt T, Lange S, Sonnenberger S, Dobner $B$, Demé B, Langner A, et al. The long periodicity phase (LPP) controversy part I: the influence of a natural-like ratio of the CEREOS analogue EOS-br in a CERNP/AP based stratum corneum modelling system: a neutron diffraction study. Biochim Biophys Acta. 2019;1861:306-15.

32 Bouwstra JA, Gooris GS, van der Spek JA, Bras W. Structural investigations of human stratum corneum by small-angle X-ray scattering. J Invest Dermatol. 1991;97:1005-12.

33 White SH, Mirejovsky D, King GI. Structure of lamellar lipid domains and corneocyte envelopes of murine stratum corneum. An Xray diffraction study. Biochemistry. 1988;27: 3725-32.

34 Bouwstra JA, Gooris GS, Bras W, Downing DT. Lipid organization in pig stratum corneum. J Lipid Res. 1995;36:685-95.

35 Bouwstra JA, Gooris GS, Weerheim AM, Kempenaar JA, Ponec M. Characterization of stratum corneum structure in reconstructed epidermis by X-ray diffraction. J Lipid Res. 1995;36:496-504.

36 Hatta I, Ohta N, Ban S, Tanaka H, Nakata S. $\mathrm{X}$-ray diffraction study on ordered, disordered and reconstituted intercellular lipid lamellar structure in stratum corneum. Biophys Chem. 2001;89:239-42.

37 Ohta N, Ban S, Tanaka H, Nakata S, Hatta I. Swelling of intercellular lipid lamellar structure with short repeat distance in hairless mouse stratum corneum as studied by X-ray diffraction. Chem Phys Lipids. 2003;123:1-8.

38 Hatta I, Ohta N, Inoue K, Yagi N. Coexistence of two domains in intercellular lipid matrix of stratum corneum. BBA. 2006;1758:1830-6.

39 Bouwstra JA, Gooris GS, Cheng K, Weerheim AM, Bras W, Ponec M. Phase behavior of isolated skin lipids. J Lipid Res. 1996;37:9991011.

40 McIntosh TJ, Stewart ME, Downing DT. Xray diffraction analysis of isolated skin lipids: reconstitution of intercellular lipid domains. Biochemistry. 1996;35:3649-53.
41 Bouwstra JA, Gooris GS, Dubbelaar FER, Ponec M. Cholesterol sulfate and calcium affect stratum corneum lipid organization over a wide temperature range. J Lipid Res. 1999; 40:2303-12.

42 Bouwstra JA, Cheng K, Gooris GS, Weerheim $\mathrm{AM}$, Ponec $\mathrm{M}$. The role of ceramides 1 and 2 in the stratum corneum lipid organisation. BBA. 1996;1300:177-86.

43 Bouwstra JA, Gooris GS, Dubbelaar FER, Ponec M. Phase behavior of stratum corneum lipid mixtures based on human ceramides: the role of natural and synthetic ceramide 1 . J Invest Dermatol. 2002;118:606-17.

44 Bouwstra JA, Dubbelaar FER, Gooris GS, Weerheim AM, Ponec M. The role of ceramide composition in the lipid organisation of the skin barrier. BBA BM. 1999;1419:12736.

45 Jager M, Gooris GS, Dolbnya IP, Bras W, Ponec M, Bouwstra JA. Novel lipid mixtures based on synthetic ceramides reproduce the unique stratum corneum lipid organization. J Lipid Res. 2004;45:923-32.

46 Mojumdar EH, Gooris GS, Bouwstra JA. Phase behavior of skin lipid mixtures: the effect of cholesterol on lipid organization. Soft Matter. 2015;11:4326-36.

47 Jager M, Gooris GS, Ponec M, Bouwstra JA: Acylceramide head group architecture affects lipid organization in synthetic ceramide mixtures. J Invest Dermatol. 2004;123:911-6.

48 Jager M, Gooris GS, Dolbnya IP, Ponec M, Bouwstra JA. Modelling the stratum corneum lipid organisation with synthetic lipid mixtures: the importance of synthetic ceramide composition. BBA. 2004;1664:132-40.

49 Masukawa Y, Narita H, Shimizu E, Kondo N, Sugai Y, Oba T, et al. Characterization of overall ceramide species in human stratum corneum. J Lipid Res. 2008;49:1466-76.

50 Norlén L. Skin barrier structure and function: the single gel phase model. J Invest Dermatol. 2001;117:830-6.

51 Norlén L. Molecular skin barrier models and some central problems for the understanding of skin barrier structure and function. Skin Pharmacol Physiol. 2003;16:203-11.

52 Boncheva M, Damien F, Normand V. Molecular organization of the lipid matrix in intact Stratum corneum using ATR-FTIR spectroscopy. BBA. 2008;1778:1344-55.

53 Norlén L. Current understanding of skin barrier morphology. Skin Pharmacol Physiol. 2013;26:213-6.

54 Garson JC, Doucet J, Lévêquê J-L, Tsoucaris G. Oriented structure in human stratum corneum revealed by X-ray diffraction. J Invest Dermatol. 1991;96:43-9.

55 Lundborg M, Narangifard A, Wennberg CL, Lindahl E, Daneholt B, Norlén L. Human skin barrier structure and function analyzed by cryo-EM and molecular dynamics simulation. J Struct Biol. 2018;203:149-61.
56 Lundborg M, Wennberg CL, Narangifard A, Lindahl E, Norlén L. Predicting drug permeability through skin using molecular dynamics simulation. J Control Release. 2018;283: 269-79.

57 Norlén L. Skin barrier structure, function and formation: learning from cryo-electron microscopy of vitreous, fully hydrated native human epidermis. Int J Cosmet Sci. 2003;25: 209-26.

58 Iwai I, Han H, den Hollander L, Svensson S, Ofverstedt L-G, Anwar J, et al. The human skin barrier is organized as stacked bilayers of fully extended ceramides with cholesterol molecules associated with the ceramide sphingoid moiety. J Invest Dermatol. 2012; 132:2215-25.

59 Skolova B, Hudska K, Pullmannova P, Kováčik A, Palát K, Roh J, et al. Different phase behavior and packing of ceramides with long (C16) and very long (C24) acyls in model membranes: infrared spectroscopy using deuterated lipids. J Phys Chem B. 2014;118: 10460-70.

60 Forslind B. A domain mosaic model of the skin barrier. Acta Derm Venereol. 1994;74: $1-6$.

61 Forslind B, Engström S, Engblom J, Norlén L. A novel approach to the understanding of human skin barrier function. J Dermatol Sci. 1997;14:115-25.

62 Bouwstra JA, Ponec M. The skin barrier in healthy and diseased state. BBA. 2006;1758: 2080-95.

63 Bouwstra JA, Gooris GS, Dubbelaar FER, Weerheim AM, Ijzerman AP, Ponec M. Role of ceramide 1 in the molecular organization of the stratum corneum lipids. J Lipid Res. 1998;39:186-96.

64 Kiselev MA, Ryabova NY, Balagurov AM, Dante S, Hauss T, Zbytovská J, et al. New insights into the structure and hydration of a stratum corneum lipid model membrane by neutron diffraction. Eur Biophys J. 2005;34: 1030-40.

65 Kiselev M, Ryabova N, Balagurov A, Dante S, Hauss T, Zbytovská J, et al. New insights into the structure and hydration of a stratum corneum lipid model membrane by neutron diffraction. Eur Biophys J. 2005;34:1030-40.

66 Sassa T, Kihara A. Metabolism of very longchain Fatty acids: genes and pathophysiology. Biomol Ther. 2014;22:83-92.

67 Brief E, Kwak S, Cheng JT, Kitson N, Thewalt JL, Lafleur M. Phase behavior of an equimolar mixture of N-palmitoyl-d-erythro-sphingosine, cholesterol, and palmitic acid, a mixture with optimized hydrophobic matching. Langmuir. 2009;25:7523-32.

68 Garidel P, Folting B, Schaller I, Kerth A. The microstructure of the stratum corneum lipid barrier: mid-infrared spectroscopic studies of hydrated ceramide:palmitic acid:cholesterol model systems. Biophys Chem. 2010;150: $144-56$. 
69 Oguri M, Gooris GS, Bito K, Bouwstra JA. The effect of the chain length distribution of free fatty acids on the mixing properties of stratum corneum model membranes. BBA. 2014; 1838:1851-61.

70 Chen H-C, Mendelsohn R, Rerek ME, Moore DJ. Effect of cholesterol on miscibility and phase behavior in binary mixtures with synthetic ceramide 2 and octadecanoic acid. Infrared studies. BBA. 2001;1512:345-56.

71 Badhe Y, Gupta R, Rai B. Structural and barrier properties of the skin ceramide lipid bilayer: a molecular dynamics simulation study. J Mol Model. 2019;25:140.

72 Gooris GS, Bouwstra JA. Infrared spectroscopic study of stratum corneum model membranes prepared from human ceramides, cholesterol, and fatty acids. Biophys J. 2007;92:2785-95.

73 Čuř́ková-Kindlová BA, Diat O, Štěpánek F, Vávrová K, Zbytovská J. Probing the interactions among sphingosine and phytosphingosine ceramides with non- and alpha-hydroxylated acyl chains in skin lipid model membranes. Int J Pharm. 2019;563:384-94.

74 Jansen LH, Hojyo-Tomoko MT, Kligman AM. Improved fluorescence staining technique for estimating turnover of the human stratum corneum. Br J Dermatol. 1974;90:912.

75 Johannesson A, Hammar H. Measurement of the horny layer turnover after staining with dansyl chloride: description of a new method. Acta Derm Venereol. 1978;58:76-9.

76 Finlay AY, Marshall RJ, Marks R. A fluorescence photographic photometric technique to assess stratum corneum turnover rate and barrier function in vivo. Br J Dermatol. 1982; 107:35-41.

77 Mojumdar EH, Helder RWJ, Gooris GS, Bouwstra JA. Monounsaturated fatty acids reduce the barrier of stratum corneum lipid membranes by enhancing the formation of a hexagonal lateral packing. Langmuir. 2014; 30:6534-43.

78 Caussin J, Gooris GS, Janssens M, Bouwstra JA. Lipid organization in human and porcine stratum corneum differs widely, while lipid mixtures with porcine ceramides model human stratum corneum lipid organization very closely. BBA. 2008;1778:1472-82.

79 Pilgram GS, Engelsma-van Pelt AM, Bouwstra JA, Koerten HK. Electron diffraction provides new information on human stratum corneum lipid organization studied in relation to depth and temperature. J Invest Dermatol. 1999;113:403-9.

80 Chen H-C, Mendelsohn R, Rerek ME, Moore DJ. Fourier transform infrared spectroscopy and differential scanning calorimetry studies of fatty acid homogeneous ceramide 2 . BBA. 2000;1468:293-303.

81 Groen D, Poole DS, Gooris GS, Bouwstra JA. Is an orthorhombic lateral packing and a proper lamellar organization important for the skin barrier function? BBA. 2011;1808: 1529-37.
82 Uche LE, Gooris GS, Beddoes CM, Bouwstra JA. New insight into phase behavior and permeability of skin lipid models based on sphingosine and phytosphingosine ceramides. Biochim Biophys Acta Biomembr. 2019;1861: 1317-28.

83 Damien F, Boncheva M. The extent of orthorhombic lipid phases in the stratum corneum determines the barrier efficiency of human skin in vivo. J Invest Dermatol. 2010;130:6114

84 Lingwood D, Simons K. Lipid rafts as a membrane-organizing principle. Science. 2010; 327:46-50.

85 Silva LC, Almeida RF de, Castro BM, Fedorov A, Prieto M. Ceramide-domain formation and collapse in lipid rafts: membrane reorganization by an apoptotic lipid. Biophys J. 2007;92:502-16.

86 Sochorová M, Audrlická P, Červená M, Kováčik A, Kopečná M, Opálka L, et al. Permeability and microstructure of cholesteroldepleted skin lipid membranes and human stratum corneum. J Colloid Interf Sci. 2019; $535: 227-38$

87 Bjorklund S, Nowacka A, Bouwstra JA, Sparr E, Topgaard D. Characterization of stratum corneum molecular dynamics by naturalabundance 13C solid-state NMR. PLoS One. 2013;8:e61889.

88 Gupta R, Rai B. Molecular dynamics simulation study of skin lipids: effects of the molar ratio of individual components over a wide temperature range. J Phys Chem B. 2015;119: 11643-55.

89 Das C, Noro MG, Olmsted PD. Fast cholesterol flip-flop and lack of swelling in skin lipid multilayers. Soft Matter. 2014;10:7346-52.

90 Janssens M, van Smeden J, Gooris GS, Bras W, Portale G, Caspers PJ, et al. Increase in shortchain ceramides correlates with an altered lipid organization and decreased barrier function in atopic eczema patients. J Lipid Res. 2012;53:2755-66.

91 Groen D, Gooris GS, Barlow DJ, Lawrence MJ, van Mechelen JB, Demé B, et al. Disposition of ceramide in model lipid membranes determined by neutron diffraction. Biophys J. 2011;100:1481-9.

92 Schmitt T, Lange S, Dobner B, Sonnenberger $S$, Hauss T, Neubert RHH, et al. Investigation of a CER[NP] and [AP] based stratum corneum modelling membrane system: using specifically deuterated CER together with a neutron diffraction approach. Langmuir. 2017;34:1742-9.

93 Das C, Noro MG, Olmsted PD. Simulation studies of stratum corneum lipid mixtures. Biophys J. 2009;97:1941-51.

94 Das C, Olmsted PD. The physics of stratum corneum lipid membranes. Philos Trans A Math Phys Eng Sci. 2016;374.

95 Mojumdar EH, Groen D, Gooris GS, Barlow DJ, Lawrence MJ, Demé B, et al. Localization of cholesterol and fatty acid in a model lipid membrane: a neutron diffraction approach. Biophys J. 2013;105:911-8.
96 Hoopes MI, Noro MG, Longo ML, Faller R Bilayer structure and lipid dynamics in a model stratum corneum with oleic acid. J Phys Chem B. 2011;115:3164-71.

97 Gruzinov AY, Zabelin AV, Kiselev MA. Short periodicity phase based on ceramide $\mathrm{AP}$ in the model lipid membranes of stratum corneum does not change during hydration. Chem Phys Lipids. 2017;202:1-5.

98 Mak VH, Potts RO, Guy RH. Does hydration affect intercellular lipid organization in the stratum corneum? Pharm Res. 1991;8:10645.

99 Schroeter A, Stahlberg S, Školová B, Sonnenberger S, Eichner A, Huster D, et al. Phase separation in ceramideNP containing lipid model membranes: neutron diffraction and solid-state NMR. Soft Matter. 2017;13: 2107-19.

100 Engelbrecht TN, Schroeter A, Hauss T, Demé B, Scheidt HA, Huster D, et al. The impact of ceramides NP and AP on the nanostructure of stratum corneum lipid bilayer. Part I: neutron diffraction and $2 \mathrm{H}$ NMR studies on multilamellar models based on ceramides with symmetric alkyl chain length distribution. Soft Matter. 2012;8:6599.

101 Sonnenberger S, Eichner A, Schmitt T, Hau $T$, Lange S, Langner A, et al. Synthesis of specific deuterated derivatives of the long chained stratum corneum lipids [EOS] and [EOP] and characterization using neutron scattering. J Labelled Comp Radiopharm. 2017;60:316-30.

102 Schmitt T, Gupta R, Lange S, Sonnenberger S, Dobner B, Hauß T, et al. Impact of the ceramide subspecies on the nanostructure of stratum corneum lipids using neutron scattering and molecular dynamics simulations. Part I: impact of CER[NS]. Chem Phys Lipids. 2018;214:58-68.

103 Garidel P. Molecular association of phytosphingosine ceramides of type III as investigated by synchrotron X-ray: part 1. HASYLAB Ann Rep. 2005:1053-4.

104 Bouwstra JA, Gooris GS, van der Spek JA, Lavrijsen SP, Bras W. The lipid and protein structure of mouse stratum corneum: a wide and small angle diffraction study. BBA. 1994;1212:183-92.

105 Swartzendruber DC, Wertz PW, Kitko DJ, Madison KC, Downing DT. Molecular models of the intercellular lipid lamellae in mammalian stratum corneum. J Invest Dermatol. 1989;92:251-7.

106 Kováčik A, Opálka L, Šilarová M, Roh J, Vávrová K. Synthesis of 6-hydroxyceramide using ruthenium-catalyzed hydrosilylationprotodesilylation. Unexpected formation of a long periodicity lamellar phase in skin lipid membranes. RSC Adv. 2016;6:73343-50.

107 Kováčik A, Vogel A, Adler J, Pullmannová P, Vávrová K, Huster D. Probing the role of ceramide hydroxylation in skin barrier lipid models by $2 \mathrm{H}$ solid-state NMR spectroscopy and X-ray powder diffraction. Biochim Biophys Acta Biomembr. 2018;1860:1162-70. 
108 Kessner D, Kiselev MA, Hauss T, Dante S, Wartewig S, Neubert RHH. Localisation of partially deuterated cholesterol in quaternary SC lipid model membranes: a neutron diffraction study. Eur Biophys J. 2008;37:10517.

109 Mojumdar EH, Gooris GS, Groen D, Barlow DJ, Lawrence MJ, Demé B, et al. Stratum corneum lipid matrix: location of acyl ceramide and cholesterol in the unit cell of the long periodicity phase. BBA. 2016;1858: 1926-34.

110 Höltje M, Förster T, Brandt B, Engels T, Rybinski W, Höltje HD. Molecular dynamics simulations of stratum corneum lipid models: fatty acids and cholesterol. BBA. 2001; 1511:156-67.

111 Das C, Noro MG, Olmsted PD. Lamellar and inverse micellar structures of skin lipids: effect of templating. Phys Rev Lett. 2013;111: 148101.

112 Sovova Z, Berka K, Otyepka M, Jurecka P. Coarse-grain simulations of skin ceramide NS with newly derived parameters clarify structure of melted phase. J Phys Chem B. 2015;119:3988-98.

113 De Sousa Neto D, Gooris GS, Bouwstra JA. Effect of the omega-acylceramides on the lipid organization of stratum corneum mod- el membranes evaluated by X-ray diffraction and FTIR studies (Part I). Chem Phys Lipids. 2011;164:184-95.

114 Grotenhuis E ten, Demel RA, Ponec M, Boer DR, van Miltenburg JC, Bouwstra JA. Phase behavior of stratum corneum lipids in mixed Langmuir-Blodgett monolayers. Biophys J. 1996;71:1389-99.

115 Masukawa Y, Narita H, Sato H, Naoe A, Kondo N, Sugai Y, et al. Comprehensive quantification of ceramide species in human stratum corneum. J Lipid Res. 2009;50: 1708-19.

116 Dahlén B, Pascher I. Molecular arrangements in sphingolipids. Thermotropic phase behaviour of tetracosanoylphytosphingosine. Chem Phys Lipids. 1979;24:119-33.

117 van Smeden J, Boiten WA, Hankemeier T, Rissmann R, Bouwstra JA, Vreeken RJ. Combined LC/MS-platform for analysis of all major stratum corneum lipids, and the profiling of skin substitutes. BBA. 2014; 1841:70-9.

118 Guo S, Moore TC, Iacovella CR, Strickland LA, McCabe C. Simulation study of the structure and phase behavior of ceramide bilayers and the role of lipid head group chemistry. J Chem Theory Comput. 2013;9:511626.
119 Moore DJ, Rerek ME, Mendelsohn R. FTIR spectroscopy studies of the conformational order and phase behavior of ceramides. J Phys Chem B. 1997;101:8933-40.

120 Moore DJ, Rerek ME, Mendelsohn R. Role of ceramides 2 and 5 in the structure of the stratum corneum lipid barrier. Int J Cosmet Sci. 1999;21:353-68.

121 Novotny J, Janusova B, Novotny M, Hrabálek A, Vávrová K. Short-chain ceramides decrease skin barrier properties. Skin Pharmacol Physiol. 2009;22:22-30.

122 Paloncyova M, Vavrova K, Sovova Z, DeVane R, Otyepka M, Berka K. Structural changes in ceramide bilayers rationalize increased permeation through Stratum Corneum models with shorter acyl tails. J Phys Chem B. 2015;119:9811-9.

123 Skolova B, Janusova B, Vavrova K. Ceramides with a pentadecasphingosine chain and short acyls have strong permeabilization effects on skin and model lipid membranes. Biochim Biophys Acta. 2016;1858:220-32.

124 Elias PM. The importance of epidermal lipids for the stratum corneum barrier. In: Osborne DW, Amann AH, editors. Topical drug delivery formulations. Drugs and the pharmaceutical sciences. New York: M. Dekker; 1990. Vol. 42; p. 13-28. 